\title{
Ist Cognitive Behavioral Analysis System of Psychotherapy (CBASP) eine Verhaltenstherapie?
}

\author{
Katrin Wambach Winfried Rief \\ Psychotherapie-Ambulanz Marburg am Fachbereich Psychologie der Philipps-Universität Marburg, Deutschland
}

Die Vielzahl neuer Verfahren auf dem Therapiemarkt macht die Zuordnung der Ansätze zu den traditionellen Therapieschulen immer unübersichtlicher. Insbesondere die Verhaltenstherapie (VT) entwickelt sich scheinbar wellenartig weiter und die neuen Ansätze wie Dialektisch-Behaviorale Therapie (DBT) [Linehan, 1993], Acceptance and Commitment Therapy (ACT) [Hayes et al., 1999]; Schematherapie (ST) [Young et al., 2003], Mindfulness Based Cognitive Therapy (MBCT) [Segal et al., 2002], Metacognitive Therapy (MCT) [Wells, 2000] und Cognitive Behavioral Analysis System of Psychotherapy (CBASP) [McCullough, 2000] fordern uns hinsichtlich der Einordnung zu übergeordneten Therapierichtungen heraus. Aus diesem Grund wollen wir das letztgenannte Verfahren CBASP einem Praxistest in Sachen VT unterziehen, mit dem Ziel, diese Therapieform näher einzuordnen.

Hinter dem sperrigen Akronym CBASP verbirgt sich ein bemerkenswertes Psychotherapieverfahren, das vom USamerikanischen Psychologen James McCullough [McCullough, 2000; deutsche Version: McCullough, 2006] speziell zur Behandlung von Patienten mit chronischer Depression entwickelt wurde. Da seine Entwicklung deutlich vor der 3. Welle der VT begann, und für eine spezifische Störungsgruppe entwickelt wurde, ist es in der Tradition der störungsorientierten Therapieansätze der letzten Jahrzehnte zu sehen. Den theoretischen Hintergrund bilden unter anderem Piagets Theorie der kognitiv-emotionalen Entwicklung, Pawlows Modell des respondenten Lernens, Skinners Erkenntnisse zum operanten Lernen, Seligmanns Modell der erlernten Hilflosigkeit sowie Kieslers Modell zur Interpersonellen Theorie. Dies mutet zunächst wie ein bunter Mix aus einflussreichen psychologischen Theorien an, ist von McCullough jedoch für das Störungs- und Behandlungsmodell sehr stringent miteinander verknüpft worden. «Die Psychopathologie des Patienten mit früh beginnender chronischer Depression wird durch anhaltende interpersonelle Vermeidung und eine
Wahrnehmungsentkoppelung von der eigenen interpersonellen Umwelt aufrechterhalten.» [McCullough, 2013]. Folgerichtig stellt CBASP die Interaktion innerhalb des sozialen und interpersonellen Umfelds in den Mittelpunkt der Behandlung. Dazu werden die Auswirkungen «prägender» Beziehungserfahrungen erarbeitet, um die Entstehung der Störung und interpersoneller Schwierigkeiten nachzuvollziehen und z.B. in der therapeutischen Beziehung für korrigierende Lern- und Reifungsprozesse einzusetzen (interpersonelle Diskriminationsübungen) [McCullough, 2012]. Weiterhin erfolgt eine konkrete Analyse problembehafteter interpersoneller Situationen aus dem Alltag des Patienten (Situationsanalysen). Im Verlauf der Therapie ergänzen sich das Durcharbeiten von Situationsanalysen (inklusive nachfolgendem Rollenspiel zur Erprobung des Zielverhaltens) und die interpersonellen Übungen innerhalb der Therapiesituation. Die Themen sind hierbei individuell an Problematik und die jeweils schwierigen Situationen des Patienten angepasst. Bei optimal verlaufender Therapie lernt der Patient, interaktionelle Situationen besser wahrzunehmen, formal-operatorisches Denken in Problemsituationen einzusetzen, Effekte des eigenen Verhaltens wahrzunehmen sowie maladaptives Verhalten zu erkennen und selbst zu korrigieren.

Um zu prüfen, inwieweit CBASP nun VT ist, orientieren wir uns an der Definition von VT nach Margraf [Margraf und Schneider, 2009]. CBASP basiert auf empirischer Psychologie und integriert eine Reihe von bewährten, teils experimentell belegten Modellen der Psychologie zu grundlegenden psychologischen Prozessen und deren Veränderung (Piaget, Pawlow, Skinner). Eine Besserung der behandelten Symptomatik wird auf verschiedenen Ebenen direkt angestrebt (Wahrnehmung, Verhalten, Denken) und eingeübt (Situationsanalyse inklusive Fertigkeitentraining am Ende). Die Störungsdiagnostik ist vor allem hinsichtlich der korrekten Einordnung der Depression und ihres Verlaufs vorgesehen, eine weitere Stö-

\section{KARGER \\ Fax +497614520714 \\ Information@Karger.de}

www.karger.com (c) 2012 S. Karger GmbH, Freiburg

1016-6262/12/0224-0224\$38.00/0

Accessible online at:

www.karger.com/ver
Dr. Katrin Wambach

Psychotherapie-Ambulanz Marburg

am Fachbereich Psychologie der Philipps-Universität Marburg

Gutenbergstraße 18,35032 Marburg, Deutschland

wambach@uni-marburg.de 
rungsdiagnostik kann problemlos ergänzt werden. Eine individuelle Problemanalyse erfolgt sehr konsequent, sowohl hinsichtlich der Entstehungsgeschichte als auch zu einzelnen Problemsituationen (Situationsanalysen). CBASP hat sich seit seiner Entstehung weiterentwickelt [McCullough, 2012] und wird zudem in weiteren Behandlungskontexten angewandt [z.B. Schramm, 2012; Cukrowicz und Joiner, 2007]. Als empirischer Beleg liegt derzeit eine große randomisierte und kontrollierte Studie vor, die aber lediglich die Wirksamkeit im Vergleich zu einem Medikament (Nefazodon, das inzwischen wegen seiner Nebenwirkungen nicht mehr auf dem Markt ist) nachweist. In beiden Gruppen profitieren etwa $50 \%$ von der Behandlung (12 Wochen, 16-20 Sitzungen); am wirksamsten ist jedoch die Kombination von Antidepressivum und CBASP. Hier zeigten $85 \%$ der Teilnehmer eine deutliche Besserung der depressiven Symptome [Keller et al., 2000]. Traumatisierte Patienten sprachen dabei besser auf CBASP an [Nemeroff et al., 2003]. Allerdings liegt auch eine Studie vor, in der CBASP die Effektivität einer individuell augmentierten Pharmakotherapie nicht veränderte und in der Wirkung vergleichbar mit einer Gruppe war, die supportive Therapie erhalten hatte [Kocsis et al., 2009]. Wie auch bei den anderen neueren Verfahren wären weitere Effektivitätsnachweise zur besseren Einschätzung erforderlich [Öst, 2008]. Dies betrifft sowohl die Modellannahmen, Merkmale der spezifischen Treatment-Integrität [Weck et al., 2011] als auch die Wirksamkeit des Verfahrens.

Auch wenn der Name dies nahelegt, ist CBASP keinesfalls nur eine Variante eines kognitiv-verhaltenstherapeutischen Verfahrens. Es ist ein Therapieverfahren mit klarer lern- und entwicklungstheoretischer Begründung, und es integriert bekannte Störungsmodelle und -mechanismen konsequent für chronisch depressive Patienten. CBASP konzeptualisiert die chronische Depression weit über das Becksche Modell [Beck, 1967] der Depression als Folge dysfunktionalen Denkens hinaus. CBASP begreift Depression als eine umfassendere Störung des Erlebens und Verhaltens und berücksichtigt in der Umset- zung das teils durch die Störung, teils durch Lernerfahrung eingeschränkte kognitive Leistungsniveau dieser Patientengruppe. Weiterhin werden Schulen übergreifend Therapieelemente ergänzt (Übertragungskonzept aus der Psychoanalyse) und die interpersonelle Problematik in den Mittelpunkt gestellt. Selten gibt es innerhalb der VT derart klare Anweisungen für die Arbeit mit der therapeutischen Beziehung. Diese konkrete Anleitung, mit negativen Gefühlen und Übertragungsphänomenen umzugehen, ist für die VT eine Bereicherung.

Auch finden Lernprinzipien und Lerngeschichte konsequenter Anwendung als innerhalb der VT selbst. Innerhalb der Übertragungs- und Beziehungsarbeit (interpersonelle Diskriminationsübung) findet Lernen im Sinne von Diskriminationslernen und Gegenkonditionierung statt [Neudeck et al., 2012]. Situationsanalysen sind als Intervention nah an den Verhaltensanalysen, aber noch klarer definiert, und sie haben den Vorteil eines strukturierten Lösungsteils, in dem Fertigkeiten individuell angepasst trainiert werden können. Dies trägt im Sinne von Problembewältigung als Wirkfaktor zum Therapieerfolg bei [Beitrag in diesem Heft: Sander et al., 2012].

Insgesamt beinhaltet CBASP eine sehr konsequente Umsetzung verhaltenstherapeutischer Prinzipien, was als Rückbesinnung aber auch als Weiterentwicklung angesehen werden kann. Von McCullough selbst wird CBASP aber in Abgrenzung zu kognitiven Verfahren (intrapsychisches Modell) als interpersoneller Ansatz bezeichnet (Person $\times$ Umwelt). CBASP hat so gesehen einen falschen Namen bekommen («misnomer»), da die interpersonelle Sichtweise viel stärker im Vordergrund steht als die kognitive. Die Integration Schulen übergreifender Behandlungselemente in CBASP hilft vielleicht, in größerem Maß die überholten Schulenstreitigkeiten zu überwinden. Weitere Nachweise der Wirksamkeit, insbesondere der direkte Vergleich mit Kognitiver Verhaltenstherapie, stehen jedoch noch aus. Dazu wird jedoch schon eine Reihe von Studien durchgeführt [Wiersma et al., 2008; Schramm et al., 2011] und geplant, deren Ergebnisse mit Spannung erwartet werden.

\section{Literatur}

Beck AT: Depression. New York, Harper and Row, 1967.

Cukrowicz KC, Joiner TE: Computer-based intervention for anxious and depressive symptoms in a nonclinical population. Cog Ther Res 2007;31:677-693.

Hayes SC, Strosahl KD, Wilson KG: Acceptance and Ccommitment Therapy: An Experiential Approach to Behavior Change. New York, Guilford Press, 1999.

Keller MB, McCullough JP, Klein DN, Arnow B, Dunner DL, Gelenberg AJ, Markowitz JC, Nemeroff CB, Russell JM, Thase ME, Trivedi MH, Zajecka J: A comparison of nefazodone, the cognitive behavioral-analysis system of psychotherapy, and their combination for the treatment of chronic depression. N Engl J Med 2000;342:1462-1470.
Kocsis JH, Gelenberg AJ, Rothbaum BO, Klein DN, Trivedi MH, Manber R, Keller MB Leon AC, Wisniewski SR, Arnow BA, Markowitz JC, Thase ME, REVAMP Investigators: Cognitive behavioral analysis system of psychotherapy and brief supportive psychotherapy for augmentation of antidepressant nonresponse in chronic depression: the REVAMP Trial. Arch Gen Psychiatry 2009;66:11781188.

Linehan MM: Cognitive-Behavioral Treatment of Borderline Personality Disorder. New York, Guilford Press, 1993.

Margraf J: Hintergründe und Entwicklung; in Margraf J, Schneider S (eds): Lehrbuch der Verhaltenstherapie. Bd 1: Grundlagen, Diagnostik, Verfahren, Rahmenbedingungen. Berlin, Springer, 2009.
McCullough JP: Treatment for Chronic Depression: Cognitive Behavioral Analysis System of Psychotherapy (CBASP). New York, Guilford Press, 2000 McCullough JP: Psychotherapie der chronischen Depression. Cognitive Behavioral Analysis System of Psychotherapy (CBASP). München, Elsevier, 2006.

McCullough JP: Therapeutische Beziehung und die Behandlung chronischer Depressionen. Heidelberg, Springer, 2012.

McCullough JP: CBASP in der Praxis: Grundlegende Konzepte und neue Entwicklungen; in Belz M, Caspar F, Schramm E. (eds): CBASP in der Praxis. München, Elsevier, 2013 (im Druck). 
Nemeroff CB, Heim CM, Thase ME, Klein DN, Rush AJ, Schatzberg AF, Ninan PT, McCullough JP Jr, Weiss PM, Dunner DL, Rothbaum BO, Kornstein S, Keitner G, Keller MB: Differential responses to psychotherapy versus pharmacotherapy in patients with chronic forms of major depression and childhood trauma. Proc Nat Acad Sci USA 2003;100: 14293-14296.

Neudeck P, Walter H, Schoepf D: Exposure aspects of the interpersonal discrimination exercise (IDE) and the situational analysis (SA) in cognitive behavioral analysis system of psychotherapy (CBASP); in Neudeck, P (ed): Exposure Therapy: Rethinking the Model - Refining the Method. New York, Springer 2012, pp 153-166.

Öst LG: Efficacy of the third wave of behavioral therapies: a systematic review and meta-analysis. Behay Res Ther 2008;46:296-321.
Sander L, Zobel I, Dykierek P, Schramm E: Allgemeine Wirkfaktoren bei der Behandlung chronisch depressiver Patienten. Vergleich zweier Psychotherapieansätze. Verhaltenstherapie;22:228-235.

Schramm E, Hautzinger M, Zobel I, Kriston L, Berger M, Härter M: Comparative efficacy of the cognitive behavioral analysis system of psychotherapy versus supportive psychotherapy for early onset chronic depression: design and rationale of a multisite randomized controlled trial. BMC Psychiatry 2011:11:134. Schramm E: CBASP in der Gruppe. Das Kurzmanual. Cognitive Behavioral Analysis System of Psychotherapy (CBASP) For Groups. The Brief Manual. Stuttgart, Schattauer, 2012.

Segal ZV, Williams, JMG, Teasdale, JD: MindfulnessBased Cognitive Therapy for Depression: A New Approach to Preventing Relapse. New York, Guilford Press, 2002.
Weck F, Bohn C, Ginzburg DM, Stangier U: Behandlungsintegrität: Implementierung, Messung, Evaluation und Zusammenhänge zum Therapieerfolg. Verhaltenstherapie 2011;21:99-108.

Wells A: Emotional Disorders and Metacognition: Innovative Cognitive Therapy. New York, John Wiley and Sons, 2000.

Wiersma JE, van Schaik DJ, van Oppen P, McCullough JP, Schoevers RA, Dekker JJ, Blom, MB, Maas K, Smit JH, Penninx BW, Beekman AT: Treatment of chronically depressed patients: a multisite randomized controlled trial testing the effectiveness of «Cognitive Behavioral Analysis System of Psychotherapy» (CBASP) for chronic depressions versus usual secondary care. BMC Psychiatry 2008;8:18

Young JE, Klosko, JS, Weishaar, ME: Schema Therapy: A Practitioner's Guide. New York, Guilford Press, 2003. 